\title{
Significado prognóstico dos graus histológicos do linfoma de Hodgkin do tipo esclerose nodular
}

\author{
Prognostic significance of histopathological grading of nodular sclerosing Hodgkin's lymphoma
}

Luís Fernando Pracchia', Valeria Buccheri², Yara de Menezes ${ }^{3}$, Sheila A. C. Siqueira ${ }^{3}$, Nair Sumie Mori', Dalton Alencar Fisher Chamone ${ }^{4}$

\section{unitermos Linfoma de Hodgkin/patologia}

Prognóstico

Análise de sobrevivência

\section{resumo}

Introdução: A esclerose nodular (EN), do tipo histológico freqüente do linfoma de Hodgkin (LH), apresenta grande variabilidade em sua composição celular. Na década de 80 , pesquisadores do British National Lymphoma Investigation (BNLI) propuseram uma subclassificação histológica do LH EN. Eles identificaram dois graus histológicos - o LH EN grau I (LH EN I) e o LH EN grau II (LH EN II) - e demonstraram que os portadores de LH EN II apresentavam menor sobrevida em comparação aos portadores de LH EN I. Outros estudos, entretanto, não reproduziram esses achados. Objetivo: Avaliar o significado prognóstico da graduação histológica proposta pelo BNLI. Materiais e métodos: Estudo retrospectivo que incluiu 69 casos de LH EN. Mais de $90 \%$ dos casos foram tratados com terapia combinada ou quimioterapia exclusiva, não havendo diferença no tipo de tratamento oferecido a depender do grau histológico. Resultados: Trinta e cinco casos (51\%) foram classificados com EN I e 34 (49\%) como EN II. Não observamos diferenças na distribuição de outros fatores prognósticos entre os portadores dos dois graus. Remissão completa após o tratamento inicial foi obtida em $85,7 \%$ dos casos de EN I e em $82,4 \%$ dos casos de EN II $(p=0,75)$. A probabilidade estimada de sobrevida global em cinco anos foi de $67 \%$ para EN I e de $83,5 \%$ para EN II $(p=0,13)$ e a taxa de sobrevida livre de doença em cinco anos foi de $85,2 \%$ versus $87 \%$, respectivamente $(p=0,72)$. Conclusão: Nesta população de pacientes uniformemente tratados a graduação histológica BNLI não esteve associada com o prognóstico do LH EN. abstract

Background: Nodular sclerosis (NS), a frequent histological subtype of Hodgkin's lymphoma (HL), presents great variability in its cellular composition. In the 80's, researchers of the British National Lymphoma Investigation (BNLI) proposed a histological-based subclassification for NS HL. They identified two histological grades - NS HL grade I (NS HL I) and NS HL grade II (NS HL II) - and reported that NS II patients had a lower survival rate in comparison with NS I patients. Others studies, however, did not reproduce these findings. Objective: Evaluate the prognostic significance of the BNLI grading system. Material and method: We retrospectively studied 69 NS HL patients. More than $90 \%$ of the included cases were treated with combined modality therapy or exclusive chemotherapy, the treatment type was not different for NS I and NS II patients. Results: Thirty-five cases (51\%) were classified as NS I and 34 (49\%) as NS II. We did not observe significant differences in the distribution of other prognostic factors between the two NS grades. The complete remission rate after initial therapy was $85.7 \%$ in the NS I group and $82.4 \%$ in the NS II group $(\mathrm{p}=0.75)$. The predicted 5 -year overall survival rate was $67 \%$ in the NS I patients and $83.5 \%$ in the NS II patients $(\mathrm{p}=0.13)$. The predicted 5 -year disease free survival rate for NSI and NSII patients was $85.2 \%$ and $87 \%$, respectively $(p=0.72)$. Conclusion: The histological BNLI grading system was not associated with the prognosis of this uniformly treated NS LH population. key words

Hodgkin's lymphoma/

pathology

Prognosis

Survival analysis

\footnotetext{
1. Médicos do Serviço de Hematologia do Hospital das Clínicas da Faculdade de Medicina da Universidade de São Paulo (HCFMUSP).

2. Doutora em hematologia; médica do Serviço de Hematologia do HCFMUSP.

3. Médicas do Serviço de Anatomia Patológica do HCFMUSP.

4. Professor titular da Disciplina de Hematologia e Hemoterapia da Faculdade de Medicina da Universidade de São Paulo (FMUSP).
} 


\section{Introdução}

A esclerose nodular (EN) é um tipo histológico freqüentemente encontrado em portadores de linfoma de Hodgkin $(\mathrm{LH})^{(7,25)}$. Morfologicamente, o LH EN pode ser diferenciado dos demais tipos devido à presença de bandas de fibrose que delimitam nódulos celulares. Esses nódulos contêm as células neoplásicas, predominantemente a variante lacunar da célula de Reed-Sternberg, e células reacionais. De acordo com a classificação de Rye ${ }^{(18)}$, a presença de fibras de colágeno, a formação de nódulos celulares e o achado de células de Reed-Sternberg ou variantes são os elementos necessários para o diagnóstico de EN. Todavia, a composição celular dos nódulos é variável, dependendo da quantidade e do tipo das células reacionais e da quantidade e pleomorfismo das células neoplásicas. Esses aspectos histomorfológicos heterogêneos propiciaram a criação de sistemas de subclassificação histológica do $\operatorname{LH} \operatorname{EN}^{(4,5,23)}$, formulados com o intuito de predizer seu prognóstico.

Em 1981, Bennet et al.(1) do British National Lymphoma Investigation (BNLI) propuseram uma divisão histológica do LH EN em dois graus distintos. Esses pesquisadores demonstraram que o LH EN grau I (LH EN I) estava presente em aproximadamente $60 \%$ dos casos e apresentava sobrevida maior que o LH EN grau II (LH EN II).

Jairam et al.(14), MacLennan et al. ${ }^{(19)} \mathrm{e}$ Wijlhuizen et al.(28) também observaram evidências de pior sobrevida global nos portadores de LN EN II em estudos retrospectivos realizados na década de 80 , nos quais o tratamento dos pacientes era baseado principalmente em radioterapia exclusiva. Durante a década de 90, possivelmente devido ao aumento da utilização de terapia combinada para o tratamento de $\mathrm{LH}$, outros estudos retrospectivos apresentaram resultados contraditó$\operatorname{rios}^{(9,13,21,26)}$, tornando incerto o significado prognóstico da graduação histológica proposta pelo BNLI.

Recentemente, dois estudos prospectivos realizados pelo European Organization for Research and Treatment of Cancer Groupe d'Etudes des Lymphomes de l'Adulte (EORTC-GELA) ${ }^{(3)}$ e pelo German Hodgkin's Lymphoma Study Group (GHLSG) ${ }^{(27)}$, nos quais um grande número de pacientes foi tratado com terapia combinada ou radioterapia nodal subtotal, não evidenciaram diferença de prognóstico entre os graus. Aparentemente, esse sistema de graduação histológica do LH EN, fator prognóstico definido há mais de trinta anos em pacientes tratados durante as décadas de 60 e 70, parece ter perdido seu valor com a introdução de terapias mais efetivas a partir do final da década de 80 em centros norte-americanos e europeus.
No Brasil, pouco se sabe sobre a freqüência e o valor prognóstico dos graus histológicos do BNLI. Apenas Souza et al.(24) avaliaram a relação entre o grau histológico e a sobrevida de 67 portadores de LH EN tratados entre 1985 e 1997. Embora não tenham observado diferença de sobrevida entre os graus da $\mathrm{EN}$, nenhum dado sobre o tipo de tratamento realizado foi apresentado e, portanto, é possível que variações do tratamento durante o período do estudo possam ter sido responsáveis por esse resultado.

Este estudo tem por objetivo avaliar o significado prognóstico da graduação histológica proposta pelo BNLI em uma população brasileira de portadores de LH EN.

\section{Casuística e métodos}

\section{Pacientes}

Foram considerados elegíveis para este estudo retrospectivo todos os pacientes com $\mathrm{LH} \mathrm{EN}$, diagnosticados e tratados no serviço de Hematologia do Hospital das Clínicas da Faculdade de Medicina da Universidade de São Paulo (HCFMUSP), no período de $1^{\circ}$ de janeiro de 1992 a 31 de dezembro de 2001. Os critérios de inclusão utilizados foram idade acima de 12 anos, soronegatividade para HIV, ausências de outra neoplasia e de tratamento oncológico prévio. Além disso, somente foram incluídos pacientes que completaram o tratamento de primeira linha proposto.

\section{Graduação histológica}

Foi realizada revisão histológica de todos os pacientes incluídos, para confirmação do diagnóstico de LH EN e para a graduação segundo os critérios do $\mathrm{BNL}\left({ }^{(1)}\right.$. A revisão foi realizada em lâminas de arquivo, coradas por hematoxilina-eosina, por um patologista que desconhecia o desfecho clínico dos pacientes.

Os critérios para classificação como EN II, conforme definição do BNLI, foram:

- mais de $25 \%$ dos nódulos celulares apresentarem depleção linfocitária reticular ou depleção linfocitária com células neoplásicas pleomórficas;

- a maioria (> 80\%) dos nódulos celulares apresentarem depleção linfocitária fibro-histiocítica;

- mais de $25 \%$ dos nódulos celulares conterem células de Reed-Sternberg de aspecto bizarro e anaplásico na ausência de áreas de depleção linfocitária. 
Dados relativos à expressão dos antígenos CD30 e CD15 nas células de Reed-Sternberg foram obtidos a partir de registros do Serviço de Anatomia Patológica do HCFMUSP. Sua expressão foi avaliada por reações imuno-histoquímicas efetuadas de acordo com protocolos institucionais próprios.

\section{Estadiamento e tratamento}

Todos os pacientes foram avaliados clinicamente e realizaram tomografias computadorizadas cervical, torácica e abdominoperineal, bem como biópsia bilateral de medula óssea. Nos casos necessários, para a complementação do estadiamento, outros exames foram adicionalmente realizados. O estadiamento de todos os pacientes foi revisado e classificado de acordo com os critérios do sistema de estadiamento Ann Arbor modificados em Cotswolds ${ }^{(17)}$.

O tratamento dos pacientes foi definido por protocolos do Serviço de Hematologia do HCFMUSP vigentes à época do diagnóstico. Em linhas gerais, os pacientes em estádio patológico IA ou IIA com apresentação supradiafragmática foram submetidos à radioterapia tipo manto, em doses que variaram de 36Gy a 40Gy. Os outros pacientes, em estádios clínicos de I a IV, foram submetidos a poliquimioterapia com duração de seis ciclos, para os pacientes em remissão completa após o quarto ciclo; ou oito ciclos, para os pacientes em remissão parcial após o quarto ciclo. Os esquemas de quimioterapia utilizados foram mecloretamina, oncovina, procarbazina e prednisona (MOPP) $)^{(6)}, \mathrm{MOPP} / \mathrm{ABV}^{(16)}$ ou adriamicina, bleomicina, vimblastina e dacarbazina $(A B V D)^{(2)}$, conforme previamente descritos. Em pacientes que apresentassem lesões bulky ao diagnóstico e obtivessem remissão completa após o tratamento quimioterápico foi administrada radioterapia no sítio da lesão bulky em doses de 30Gy a 36Gy.

Os pacientes que apresentaram falha terapêutica ou recidiva foram tratados de forma individualizada, com radioterapia, quimioterapia ou transplante de medula óssea autoplástico.

\section{Definições de resposta ao tratamento e recidiva}

- Remissão completa foi definida como o desaparecimento de todas as evidências clínico-laboratoriais e radiológicas da doença ao término do tratamento, e mantido por pelo menos quatro semanas.

- Remissão parcial foi definida como a redução clínica ou radiológica de pelo menos $50 \%$ do maior diâmetro de todas as massas tumorais preexistentes observada ao término do tratamento.
- Refratariedade foi definida como falha na obtenção de resposta parcial ao término do tratamento, enquanto progressão foi definida como o aumento do tamanho das massas ou aparecimento de novas lesões durante o tratamento ou até quatro semanas de seu término. Refratariedade e progressão foram consideradas como falha do tratamento de primeira linha.

- Recidiva foi definida, para os pacientes em remissão completa, como o aparecimento de novas massas tumorais diagnosticadas por métodos clínicos, radiológicos ou histológicos, após quatro semanas do término do tratamento inicial.

\section{Variáveis prognósticas e de tratamento para análise}

Mediante revisão de prontuários, foram obtidos dados relativos as seguintes variáveis identificadas ao diagnóstico: idade, sexo, estádio clínico e/ou patológico, presença de sintomas B, velocidade de hemossedimentação (VHS), albumina sérica, nível de hemoglobina, leucometria e linfometria. Para análise, foram separadas em categorias. Além disso, foi calculado o valor do índice prognóstico internacional (IPI) ${ }^{(10,12)}$, categorizado em dois níveis: baixo risco ( 0 a 2 fatores de risco) ou alto risco (mais de 2 fatores de risco).

Também foram obtidos dados relativos ao tratamento inicial e seus desfechos: data do início do tratamento, tipo de quimioterapia, realização de radioterapia, data do término do tratamento, resposta ao tratamento, ocorrência de recidiva e sua data, ocorrência de óbito e sua data ou ocorrência de perda de seguimento e a data do último registro em prontuário.

\section{Análise dos dados}

Os pacientes foram classificados em dois grupos, segundo o grau histológico da EN obtido após revisão: grupo I (pacientes com LH EN I) e grupo II (pacientes com LH EN II).

Os dois grupos foram comparados segundo as diversas variáveis prognósticas. No tocante à idade, como variável contínua, utilizou-se o teste não-paramétrico de MannWhitney. Para as variáveis categóricas foi utilizado o teste do qui-quadrado $\left(\chi^{2}\right)$ ou o teste exato de Fisher, quando indicado.

Sobrevida global foi definida como o tempo decorrido desde o início do tratamento até o óbito por qualquer causa. Sobrevida livre de doença foi definida, para os pacientes em remissão completa, como o tempo decorrido 
do diagnóstico de remissão até o diagnóstico de recidiva da doença. Na ausência de óbito ou recidiva, os pacientes foram censurados na data do último registro em prontuário. $\mathrm{Na}$ análise das sobrevidas global e livre de doença empregou-se o estimador, produto-limite de Kaplan-Meier ${ }^{(15)}$, com elaboração dos respectivos gráficos. A comparação univariada das curvas de sobrevida foi realizada através do teste log-rank ${ }^{(20)}$.

Todos os testes foram bicaudais com nível de significância definido em $5 \%$. Para a análise dos dados foi utilizado o software SPSS 10,0 .

\section{Resultados}

\section{Pacientes}

No período de $1^{\circ}$ de janeiro de 1992 a 31 de dezembro de 2001 foram identificados 100 casos de LH EN. Trinta e um (31\%) casos foram excluídos da análise: sete tiveram seu prontuário médico extraviado, 15 não completaram o tratamento de primeira linha proposto, três já haviam realizado tratamento prévio para $\mathrm{LH}$ e seis não possuíam material disponível para revisão histológica.

\section{Revisão histológica}

As amostras histopatológicas dos 69 pacientes incluídos foram graduadas de acordo com os critérios do BNLI, resultando em 35 (51\%) casos classificados como EN I (grupo I) e 34 (49\%) casos como EN II (grupo II). Das 34 amostras do grupo II, seis (18\%) apresentavam depleção linfocitária reticular ou pleomórfica em mais de $25 \%$ dos nódulos celulares, cinco (15\%) apresentavam depleção linfocitária fibro-histiocítica na maioria dos nódulos celulares e 23 (67\%) apresentavam células neoplásicas pleomórficas e ausência de áreas de depleção linfocitária em mais de $25 \%$ dos nódulos celulares (Figura 1). Dos 69 casos, 52 (75\%) possuíam avaliação imuno-histoquímica, 27 (52\%) foram classificados como EN I e 25 (48\%) como EN II. Dos 52 casos com imu-

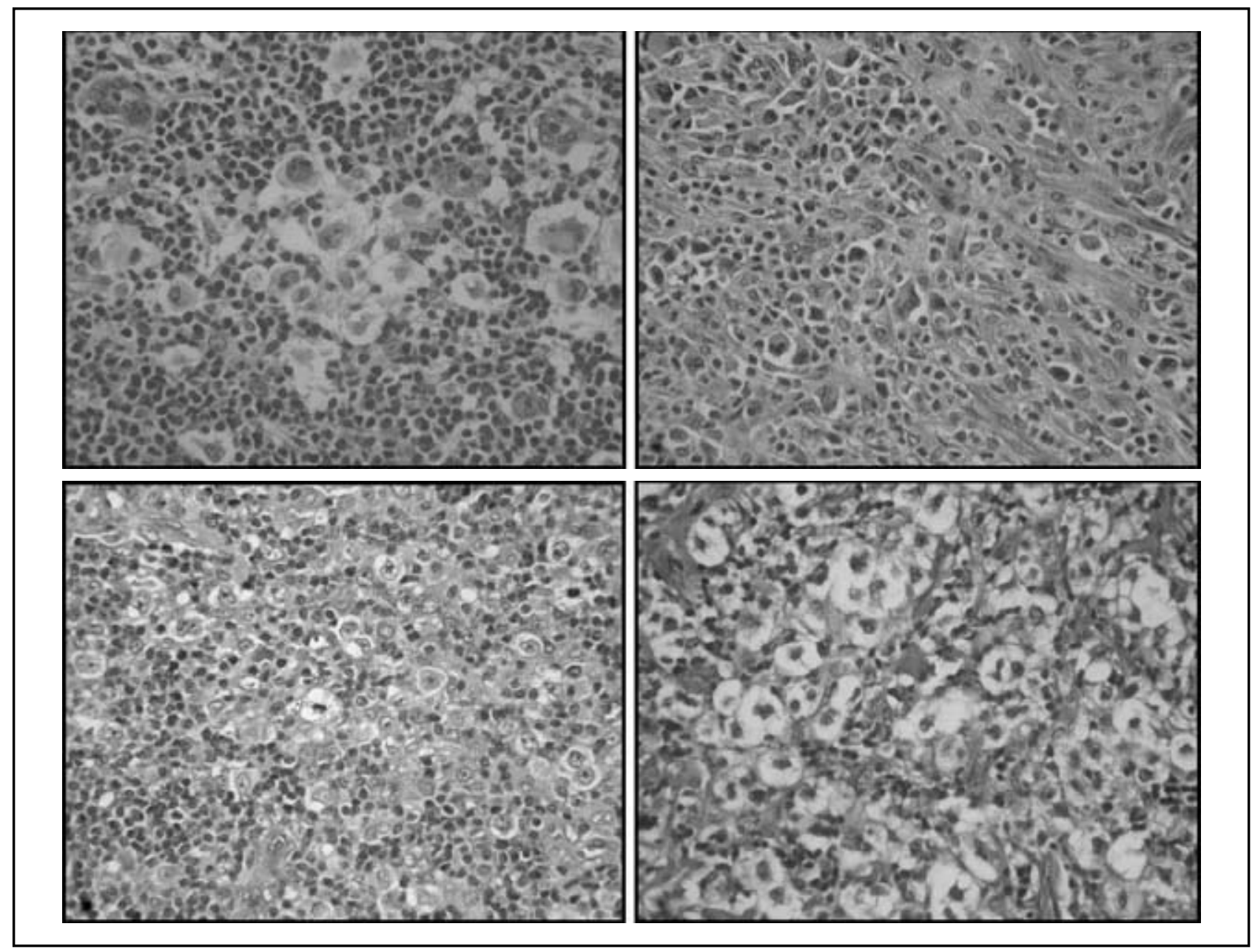

Figura 1 - Graus do linfoma de Hodgkin esclerose nodular. Detalhe dos nódulos celulares. (A) LH EN Grau I - nódulo celular com características de predominância linfocitária. (B) LH EN Grau II - nódulo celular com células de Reed-Sternberg pleomórficas. (C) LH EN Grau II - nódulo celular com características de celularidade mista/depleção linfocitária reticular. (D) LH EN Grau II - nódulo celular com características de depleção linfocitária fibro-histiocítica 
nofenótipo conhecido, 49 (94\%) apresentavam expressão de CD30, 46 (89\%) apresentavam expressão de CD15 e 43 (83\%) apresentavam expressão simultânea de CD30 e CD15. Os três casos negativos para CD30 apresentavam expressão de CD15 na ausência de CD3, CD20 e antígeno da membrana epitelial (EMA) e foram incluídos na análise.

\section{Características dos grupos}

Os dois grupos não diferiram quanto às diversas variáveis prognósticas avaliadas (Tabela 1). A mediana de idade foi 23 anos no grupo I e 25 anos no grupo II $(p=0,6)$.
A distribuição das variáveis de tratamento também foi similar entre os dois grupos (Tabela 2). Entre os 37 pacientes tratados com quimioterapia exclusiva, estão incluídos cinco pacientes do grupo I e seis do grupo II nos quais a proposta de tratamento inicial era terapia combinada, mas a radioterapia foi substituída por quimioterapia de resgate em decorrência da falta de resposta completa ao tratamento quimioterápico.

\section{Resposta ao tratamento de primeira linha}

Remissão completa ocorreu em 30 (85,7\%) dos 35 pacientes do grupo I e em 28 (82,4\%) dos 34 pacientes do grupo II

\section{Tabela 1 Características prognósticas dos grupos}

\begin{tabular}{|c|c|c|c|}
\hline Variável prognóstica & Grupo I (EN I) $n=35(\%)$ & Grupo II (EN II) $n=34(\%)$ & $p$ \\
\hline \multicolumn{4}{|l|}{ Idade } \\
\hline Até 45 anos & $33(94,3)$ & $29(85,3)$ & 0,26 \\
\hline$>45$ anos & $2(5,7)$ & $5(14,7)$ & \\
\hline \multicolumn{4}{|l|}{ Sexo } \\
\hline Masculino & $15(42,9)$ & $15(44,1)$ & 1 \\
\hline Feminino & $20(57,1)$ & $19(55,9)$ & \\
\hline \multicolumn{4}{|l|}{ Estádio } \\
\hline I/II & $22(62,9)$ & $20(58,8)$ & 0,81 \\
\hline III/IV & $13(37,1)$ & $14(41,2)$ & \\
\hline \multicolumn{4}{|l|}{ Sintomas B } \\
\hline Presentes & $20(57,1)$ & $23(67,6)$ & 0,46 \\
\hline Ausentes & $15(42,9)$ & $11(32,4)$ & \\
\hline \multicolumn{4}{|l|}{ Albumina sérica* } \\
\hline$\geq 4 \mathrm{~g} / \mathrm{dl}$ & $16(45,7)$ & $10(33,3)$ & 0,44 \\
\hline$<4 \mathrm{~g} / \mathrm{dL}$ & $19(54,3)$ & $20(66,7)$ & \\
\hline \multicolumn{4}{|l|}{ Hemoglobina } \\
\hline$\geq 10,5 \mathrm{~g} / \mathrm{dl}$ & $21(60)$ & $24(70,6)$ & 0,45 \\
\hline$<10,5 \mathrm{~g} / \mathrm{dl}$ & $14(40)$ & $10(29,4)$ & \\
\hline \multicolumn{4}{|l|}{ Leucócitos } \\
\hline$<15.000 / \mathrm{mm}^{3}$ & $27(77,1)$ & $26(76,5)$ & 1 \\
\hline$\geq 15.000 / \mathrm{mm}^{3}$ & $8(22,9)$ & $8(23,5)$ & \\
\hline \multicolumn{4}{|l|}{ Linfócitos } \\
\hline$\geq 600 / \mathrm{mm}^{3}$ & $33(94,3)$ & $31(91,2)$ & 0,67 \\
\hline$<600 / \mathrm{mm}^{3}$ & $2(5,7)$ & $3(8,8)$ & \\
\hline \multicolumn{4}{|l|}{ VHS** } \\
\hline Até $50 \mathrm{~mm} / \mathrm{h}$ & $19(61,3)$ & $16(59,3)$ & 1 \\
\hline$>50 \mathrm{~mm} / \mathrm{h}$ & $12(38,7)$ & $11(40,7)$ & \\
\hline \multicolumn{4}{|l|}{$\mathrm{IPI}^{* * *}$} \\
\hline Baixo risco & $21(60)$ & $19(55,9)$ & 0,81 \\
\hline Alto risco & $14(40)$ & $15(44,1)$ & \\
\hline
\end{tabular}

* Dado ignorado em quatro pacientes do grupo II.

** Velocidade de hemossedimentação. Dado ignorado em quatro pacientes do grupo I e sete do grupo II.

*** Índice prognóstico internacional. Baixo risco indica a presença de no máximo dois fatores de mau prognóstico. 
$(p=0,75)$. Ocorreu remissão parcial em dois $(5,9 \%)$ pacientes do grupo II, enquanto no grupo I não houve nenhum caso. Falha do tratamento de primeira linha foi observada em cinco $(14,3 \%)$ dos pacientes do grupo I e em quatro $(11,8 \%)$ do grupo II $(p=1)$. A taxa de remissão completa nos casos de imunofenótipo conhecido foi de $81,5 \%$ para o grupo I e de $80 \%$ para o grupo II $(p=0,89)$. Das características prognósticas avaliadas, apenas o IPI esteve associado de modo estatisticamente significativo com as taxas de remissão completa (Tabela 3). Dos pacientes com IPI de baixo risco, $93 \%$ obtiveram remissão completa. Por outro lado, nos com IPI de alto risco a proporção foi de $72 \%(p=0,04)$.

\section{Sobrevida global}

Em uma mediana de acompanhamento de 45 meses (variação 6 a 117 meses) foram observados 12 óbitos nos 69 pacientes incluídos: nove em pacientes do grupo I e três em pacientes do grupo II. A média do tempo de sobrevida global foi de 90 meses (intervalo de confiança [IC] 95\%: 76-105 meses) para os pacientes do grupo I e de 96 meses (IC 95\%: 85-106 meses) para os do grupo II (Figura 2). A probabilidade estimada de sobrevida global acumulada em cinco anos foi de $67 \%$ no grupo I e de $83,5 \%$ no grupo II $(p=0,13)$. Não houve diferença estatisticamente significativa na taxa de sobrevida global, quando apenas os pacientes com imunofenótipo conhecido foram incluídos na análise (66\% para EN I e $88 \%$ para EN II $[p=0,12]$ ).

Em análise univariada, apenas o IPI, a hemoglobina e a VHS estiveram associados com a sobrevida dos pacientes (Tabela 4).

\section{Sobrevida livre de doença}

Para os 58 pacientes que atingiram remissão completa após o tratamento de primeira linha, a média de sobrevida livre de doença foi de 93 meses (IC 95\%: 79-107 meses) nos pacientes do grupo I e de 86 meses (IC 95\%: 75-97 meses) nos do grupo II (Figura 3). A probabilidade estimada de sobrevida livre de doença em cinco anos foi de $85,2 \%$ no grupo I e de $87 \%$ no grupo II $(p=0,72)$. Excluindo os 17 pacientes sem avaliação imuno-histoquímica, a taxa de sobrevida livre de doença foi de $86 \%$ no grupo I e $80 \%$ no grupo II $(p=0,6)$.

Análise univariada da relação das variáveis prognósticas com a sobrevida livre de doença demonstrou que apenas os estádios avançados estiveram relacionados a maior risco de recidiva (Tabela 5).

\section{Discussão}

Embora o LH seja uma neoplasia na qual o adequado tratamento possa assegurar longa sobrevida para a maioria de seus portadores, a identificação de fatores prognósticos é necessária para a definição de estratégias terapêuticas individualizadas, permitindo a redução da toxicidade em pacientes de bom prognóstico.

Desde 1944 foram formuladas diversas propostas de classificação histológica desse tumor, com o propósito de identificação de casos com prognósticos distintos. O sistema de graduação histológica do LH EN, proposto pelo BNLI ${ }^{(1)}$ na década de 80 , apresentou resultados controversos quando utilizado por pesquisadores norte-americanos e europeus $^{(9,13,14,19,21,26,28)}$, sendo seu valor prognóstico em nossa população ainda pouco conhecido ${ }^{(24)}$.

Neste estudo avaliamos o significado prognóstico da graduação BNLI em 69 portadores de LH EN. A freqüência por nós encontrada de EN II (49\%) foi aparentemente superior àquela observada em alguns estudos internacionais ${ }^{(3,9,13,14,19,21,26,28)}$, nos quais a frequêencia de EN II variou de $20 \%$ a $34 \%$, embora uma comparação direta desses resultados não seja possível. Freqüências de EN II acima de $35 \%$ também foram observadas por Masih ${ }^{(21)}$ et al. (69\%) e Souza ${ }^{(24)}$ et al. (38\%). A razão para essa variação na freqüência de EN II entre os diferentes estudos não é conhecida, mas do mesmo modo que existe variação na freqüência dos tipos

\section{Tabela 2 Características de tratamento dos grupos}

\begin{tabular}{lccc}
\hline Variável de tratamento & Grupo I (EN I) $n=35(\%)$ & Grupo II (EN II) $n=34(\%)$ & \\
Tipo de tratamento & & & \\
Radioterapia exclusiva & $2(5,7)$ & $0(0)$ & 0,37 \\
Terapia combinada & $15(42,9)$ & $15(44,1)$ & \\
Quimioterapia exclusiva & $18(51,4)$ & $19(55,9)$ & \\
Tipo de quimioterapia & & & 0,91 \\
MOPP & $3(9,1)$ & $4(11,8)$ & \\
ABVD & $16(48,5)$ & $15(44,1)$ & \\
MOPP/ABV & $14(42,4)$ & $15(44,1)$ &
\end{tabular}


histológicos da classificação de Rye entre regiões geográficas distintas, tanto entre o Brasil e outros países quanto entre os diferentes estados brasileiros ${ }^{(22)}$, é possível que também ocorram diferenças na freqüência dos graus histológicos da $\mathrm{EN}$, dependendo do local de origem dos pacientes. Além disso, poucos autores avaliaram a reprodutibilidade desse sistema de graduação.

Nos estudos de Benett et al.(1), Jairam et al.(14) e MacLennan et al. ${ }^{(19)}$ a graduação foi realizada por dois patologistas independentes da mesma instituição, sendo observadas taxas de concordância que variaram de $88 \%$ a $95 \%$. Porém, em recente estudo multicêntrico realizado pelo EORTC-GELA ${ }^{(3)}$, no qual um painel de seis hematopatologistas de diferentes centros foi responsável pela graduação, a taxa de concordância entre observadores foi de $78 \%$. Dessa forma, apenas um estudo multicêntrico internacional poderá esclarecer se existe variação regional dos graus histológicos ou se há variação na graduação realizada em

Tabela 3 Variáveis prognósticas e taxa de remissão completa

\begin{tabular}{|c|c|c|c|}
\hline Variável prognóstica & Remissão completa $n(\%)$ & Não-remissão completa $n(\%)$ & $p$ \\
\hline \multicolumn{4}{|l|}{ Grau histológico } \\
\hline Esclerose nodular grau I & $30(85,7)$ & $5(14,3)$ & \multirow[t]{2}{*}{0,75} \\
\hline Esclerose nodular grau II & $28(82,4)$ & $6(17,6)$ & \\
\hline \multicolumn{4}{|l|}{ Idade } \\
\hline Até 45 anos & $52(83,9)$ & $10(16,1)$ & \multirow[t]{2}{*}{1} \\
\hline$>45$ anos & $6(85,7)$ & $1(14,3)$ & \\
\hline \multicolumn{4}{|l|}{ Sexo } \\
\hline Masculino & $25(83,3)$ & $5(16,7)$ & \multirow[t]{2}{*}{1} \\
\hline Feminino & $33(84,6)$ & $6(15,4)$ & \\
\hline \multicolumn{4}{|l|}{ Estádio } \\
\hline I/II & $35(83,3)$ & $7(16,7)$ & \multirow[t]{2}{*}{1} \\
\hline III/IV & $23(85,2)$ & $4(14,8)$ & \\
\hline \multicolumn{4}{|l|}{ Sintomas B } \\
\hline Presentes & $35(81,4)$ & $8(18,6)$ & \multirow[t]{2}{*}{0,52} \\
\hline Ausentes & $23(88,5)$ & $3(11,5)$ & \\
\hline \multicolumn{4}{|l|}{ Albumina sérica* } \\
\hline$\geq 4 \mathrm{~g} / \mathrm{dl}$ & $24(92,3)$ & $2(7,7)$ & \multirow[t]{2}{*}{0,17} \\
\hline$<4 \mathrm{~g} / \mathrm{dl}$ & $30(76,9)$ & $9(23,1)$ & \\
\hline \multicolumn{4}{|l|}{ Hemoglobina } \\
\hline$\geq 10,5 \mathrm{~g} / \mathrm{dl}$ & $39(86,7)$ & $6(13,3)$ & \multirow[t]{2}{*}{0,49} \\
\hline$<10,5 \mathrm{~g} / \mathrm{dl}$ & $19(79,2)$ & $5(20,8)$ & \\
\hline \multicolumn{4}{|l|}{ Leucócitos } \\
\hline$<15.000 / \mathrm{mm}^{3}$ & $47(88,7)$ & $6(11,3)$ & \multirow[t]{2}{*}{0,11} \\
\hline$\geq 15.000 / \mathrm{mm}^{3}$ & $11(68,8)$ & $5(31,3)$ & \\
\hline \multicolumn{4}{|l|}{ Linfócitos } \\
\hline$\geq 600 / \mathrm{mm}^{3}$ & $53(82,8)$ & $11(17,2)$ & \multirow[t]{2}{*}{0,58} \\
\hline$<600 / \mathrm{mm}^{3}$ & $5(100)$ & $0(0)$ & \\
\hline \multicolumn{4}{|l|}{ VHS** } \\
\hline Até $50 \mathrm{~mm} / \mathrm{h}$ & $32(91,4)$ & $3(8,6)$ & \multirow[t]{2}{*}{0,13} \\
\hline$>50 \mathrm{~mm} / \mathrm{h}$ & $17(73,9)$ & $6(26,1)$ & \\
\hline \multicolumn{4}{|l|}{ IPI * * * } \\
\hline Baixo risco & $37(92,5)$ & $3(7,5)$ & \multirow[t]{2}{*}{0,04} \\
\hline Alto risco & $21(72,4)$ & $8(27,6)$ & \\
\hline
\end{tabular}


diferentes instituições.

Neste estudo, os critérios de graduação do BNLI(1) foram rigorosamente seguidos e, tendo em vista que a população aqui relatada foi constituída de pacientes consecutivos, é pouco provável que algum vício de seleção tenha sido responsável pela freqüência de EN II por nós encontrada.

Em concordância com os estudos de Wijlhuizen et al. ${ }^{(28)}$, Ferry et al. ${ }^{(9)}$ e Hess et al. ${ }^{(13)}$, também não observamos asso- ciação estatística entre o grau histológico e a freqüência de outros fatores prognósticos conhecidos, como idade, sexo, estádio, presença de sintomas B e VHS. Embora alguns estudos publicados durante a década de 80 tenham sugerido tal associação $0^{(1,14,19)}$, nenhum deles realizou análise estatística dos dados. Além disso, demonstramos, de forma similar à von Wasielewski et al.(27), que o IPI, outro fator prognóstico validado internacionalmente, também não esteve associado

Tabela 4 Variáveis prognósticas e sobrevida global

\begin{tabular}{|c|c|c|c|}
\hline Variável prognóstica & Sobrevida média/Meses (IC 95\%) & $\mathrm{SG}^{\dagger}(\%)$ & $p$ \\
\hline \multicolumn{4}{|l|}{ Grau histológico } \\
\hline Esclerose nodular grau I & $90(76-105)$ & 67 & \multirow[t]{2}{*}{0,13} \\
\hline Esclerose nodular grau II & $96(85-106)$ & 83,5 & \\
\hline \multicolumn{4}{|l|}{ Idade } \\
\hline Até 45 anos & $100(90-110)$ & 78,3 & \multirow[t]{2}{*}{0,27} \\
\hline$>45$ anos & $79(57-102)$ & 55,6 & \\
\hline \multicolumn{4}{|l|}{ Sexo } \\
\hline Masculino & $98(73-112)$ & 74,5 & \multirow[t]{2}{*}{0,89} \\
\hline Feminino & $92(80-105)$ & 73,8 & \\
\hline \multicolumn{4}{|l|}{ Estádio } \\
\hline I/II & $99(89-109)$ & 82,6 & \multirow[t]{2}{*}{0,13} \\
\hline III/IV & $86(68-104)$ & 61 & \\
\hline \multicolumn{4}{|l|}{ Sintomas B } \\
\hline Presentes & $93(80-107)$ & 70,2 & \multirow[t]{2}{*}{0,33} \\
\hline Ausentes & $93(83-105)$ & 80,7 & \\
\hline \multicolumn{4}{|l|}{ Albumina sérica* } \\
\hline$\geq 4 \mathrm{~g} / \mathrm{dl}$ & $103(90-115)$ & 78,9 & \multirow[t]{2}{*}{0,26} \\
\hline$<4 \mathrm{~g} / \mathrm{dl}$ & $87(73-100)$ & 69 & \\
\hline \multicolumn{4}{|l|}{ Hemoglobina } \\
\hline$\geq 10,5 \mathrm{~g} / \mathrm{dl}$ & $102(94-111)$ & 87,2 & \multirow[t]{2}{*}{0,03} \\
\hline$<10,5 \mathrm{~g} / \mathrm{dl}$ & $77(56-97)$ & 49 & \\
\hline \multicolumn{4}{|l|}{ Leucócitos } \\
\hline$<15.000 / \mathrm{mm}^{3}$ & $98(87-109)$ & 73,9 & \multirow[t]{2}{*}{0,6} \\
\hline$\geq 15.000 / \mathrm{mm}^{3}$ & $90(70-110)$ & 77,6 & \\
\hline \multicolumn{4}{|l|}{ Linfócitos } \\
\hline$\geq 600 / \mathrm{mm}^{3}$ & $99(89-109)$ & 77,6 & \multirow[t]{2}{*}{0,21} \\
\hline$<600 / \mathrm{mm}^{3}$ & $59(46-71)$ & 66 & \\
\hline \multicolumn{4}{|l|}{ VHS ** } \\
\hline Até $50 \mathrm{~mm} / \mathrm{h}$ & $101(92-110)$ & 83,7 & \multirow[t]{2}{*}{0,04} \\
\hline$>50 \mathrm{~mm} / \mathrm{h}$ & $75(52-97)$ & 47,2 & \\
\hline \multicolumn{4}{|l|}{$\mathrm{IPI}^{*}$ * * } \\
\hline Baixo risco & $103(94-112)$ & 88 & \multirow[t]{2}{*}{0,01} \\
\hline Alto risco & $82(65-100)$ & 56 & \\
\hline
\end{tabular}

* Dado ignorado em quatro pacientes do grupo II.

** Velocidade de hemossedimentação. Dado ignorado em quatro pacientes do grupo I e sete do grupo II.

*** Índice prognóstico internacional. Baixo risco indica a presença de no máximo dois fatores de mau prognóstico.

† Probabilidade estimada de sobrevida global em cinco anos. 
ao grau histológico do LH EN.

A presente série, composta de pacientes diagnosticados durante a década de 90 , foi tratada de forma padronizada. Mais de $90 \%$ dos casos receberam terapia combinada (radio e quimioterapia) ou poliquimioterapia exclusiva. Radioterapia exclusiva somente foi realizada em dois pacientes em estádios patológicos IA ou IIA.

Estudos multicêntricos prospectivos ${ }^{(8,11)}$ demonstraram que os esquemas de quimioterapia MOPP-ABV e $A B V D$ apresentam eficácia similar e superior ao esquema MOPP, mas como o tipo de tratamento oferecido não diferiu entre os portadores de LH EN I ou II, é improvável que a utilização de um esquema quimioterápico menos eficaz possa ter influenciado as respostas terapêuticas aqui descritas. Com o emprego de um tratamento inicial baseado principalmente em terapia combinada ou quimioterapia, não observamos

\section{Tabela 5 Variáveis prognósticas e sobrevida livre de doença}

\begin{tabular}{|c|c|c|c|}
\hline Variável prognóstica & Sobrevida média/Meses (IC 95\%) & $\operatorname{SLD}^{\dagger}(\%)$ & $p$ \\
\hline \multicolumn{4}{|l|}{ Grau histológico } \\
\hline Esclerose nodular grau I & $93(79-107)$ & 85,2 & \multirow[t]{2}{*}{0,72} \\
\hline Esclerose nodular grau II & $86(75-97)$ & 87 & \\
\hline \multicolumn{4}{|l|}{ Idade } \\
\hline Até 45 anos & $95(85-105)$ & 87 & \multirow[t]{2}{*}{0,91} \\
\hline$>45$ anos & $84(62-107)$ & 83,3 & \\
\hline \multicolumn{4}{|l|}{ Sexo } \\
\hline Masculino & $90(73-106)$ & 80,7 & \multirow[t]{2}{*}{0,33} \\
\hline Feminino & $90(80-100)$ & 89,8 & \\
\hline \multicolumn{4}{|l|}{ Estádio } \\
\hline I/II & $96(91-102)$ & 96,8 & \multirow[t]{2}{*}{0,01} \\
\hline III/IV & $79(59-99)$ & 69 & \\
\hline \multicolumn{4}{|l|}{ Sintomas B } \\
\hline Presentes & $92(79-105)$ & 84 & \multirow[t]{2}{*}{0,49} \\
\hline Ausentes & $85(74-96)$ & 89,5 & \\
\hline \multicolumn{4}{|l|}{ Albumina sérica* } \\
\hline$\geq 4 \mathrm{~g} / \mathrm{dl}$ & $95(82-109)$ & 87 & \multirow[t]{2}{*}{0,72} \\
\hline$<4 \mathrm{~g} / \mathrm{dl}$ & $84(71-97)$ & 84,1 & \\
\hline \multicolumn{4}{|l|}{ Hemoglobina } \\
\hline$\geq 10,5 \mathrm{~g} / \mathrm{dl}$ & $92(84-100)$ & 91,9 & \multirow[t]{2}{*}{0,12} \\
\hline$<10,5 \mathrm{~g} / \mathrm{dl}$ & $82(60-104)$ & 75 & \\
\hline \multicolumn{4}{|l|}{ Leucócitos } \\
\hline$<15.000 / \mathrm{mm}^{3}$ & $92(81-103)$ & 83,8 & \multirow[t]{2}{*}{0,21} \\
\hline$\geq 15.000 / \mathrm{mm}^{3}$ & $89(79-100)$ & 93,8 & \\
\hline \multicolumn{4}{|l|}{ Linfócitos } \\
\hline$\geq 600 / \mathrm{mm}^{3}$ & $96(86-105)$ & 87,5 & \multirow[t]{2}{*}{0,64} \\
\hline$<600 / \mathrm{mm} 3$ & $51(28-75)$ & 75 & \\
\hline \multicolumn{4}{|l|}{ VHS** } \\
\hline$<50 \mathrm{~mm} / \mathrm{h}$ & $84(72-96)$ & 83,3 & \multirow[t]{2}{*}{0,93} \\
\hline$>50 \mathrm{~mm} / \mathrm{h}$ & $92(70-113)$ & 81,2 & \\
\hline \multicolumn{4}{|l|}{$\mathrm{IPI}^{*}$ * * } \\
\hline Baixo risco & $91(83-100)$ & 91,3 & \multirow[t]{2}{*}{0,24} \\
\hline Alto risco & $86(66-105)$ & 76 & \\
\hline
\end{tabular}

* Dado ignorado em quatro pacientes do grupo II.

** Velocidade de hemossedimentação. Dado ignorado em quatro pacientes do grupo I e sete do grupo II.

*** Índice prognóstico internacional. Baixo risco indica a presença de no máximo dois fatores de mau prognóstico.

$\uparrow$ Probabilidade estimada de sobrevida livre de doença em cinco anos. 


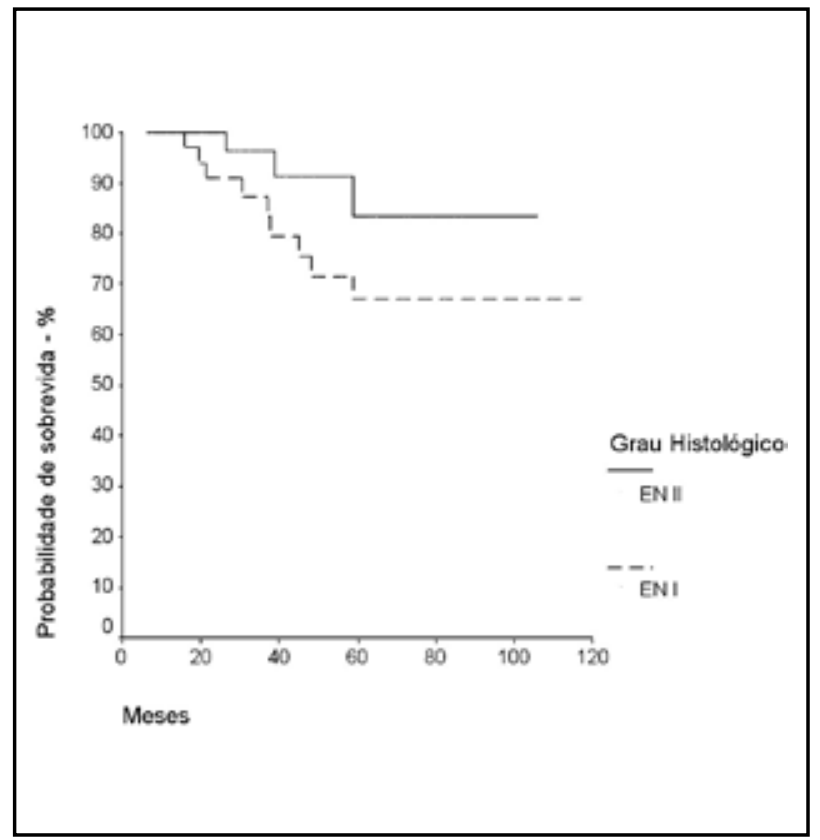

Figura 2 - Sobrevida global por grau histológico

diferenças nas taxas de resposta completa ou parcial entre os dois graus histológicos - somente o IPI esteve relacionado a menor taxa de remissão completa. Apenas dois grupos internacionais avaliaram a relação da taxa de remissão completa com o grau histológico.

MacLennan et al.(19) observaram diferença na taxa de remissão completa (83,7\% LH EN I versus $66,3 \%$ LH EN II), mas não foi realizada comparação estatística dos dados. Já Van Spronsen et al. ${ }^{(26)}$ demonstraram diferença estatisticamente significativa na taxa de remissão completa entre os dois graus histológicos (89\% LH EN I versus 73\% LH EN II, $p=0,02$ ), porém os pacientes não foram tratados de maneira uniforme e, portanto, é possível que essa diferença no tratamento oferecido tenha sido responsável pelo resultado inferior observado nos portadores de LH EN II.

Neste estudo, os fatores relacionados a menor sobrevida global dos pacientes foram o IPI, a VHS e a concentração de hemoglobina sérica ao diagnóstico. A sobrevida global não foi estatisticamente diferente entre os portadores de LH EN I e LH EN II. Resultado similar foi observado por outros autores, tanto em estudos retrospectivos nos quais terapia combinada e/ou radioterapia nodal subtotal foram utilizadas no tratamento dos pacientes ${ }^{(9,13)}$, quanto nos dois recentes estudos prospectivos realizados pelo EORTC-GELA ${ }^{(3)}$ e GHLSG ${ }^{(27)}$.

O grau histológico também não influenciou a sobrevida livre de doença dos pacientes, de forma similar aos estudos de Ferry et al. ${ }^{(9)}$ e Hess et al. ${ }^{(13)}$. Apenas MacLennan et al. ${ }^{(19)}$

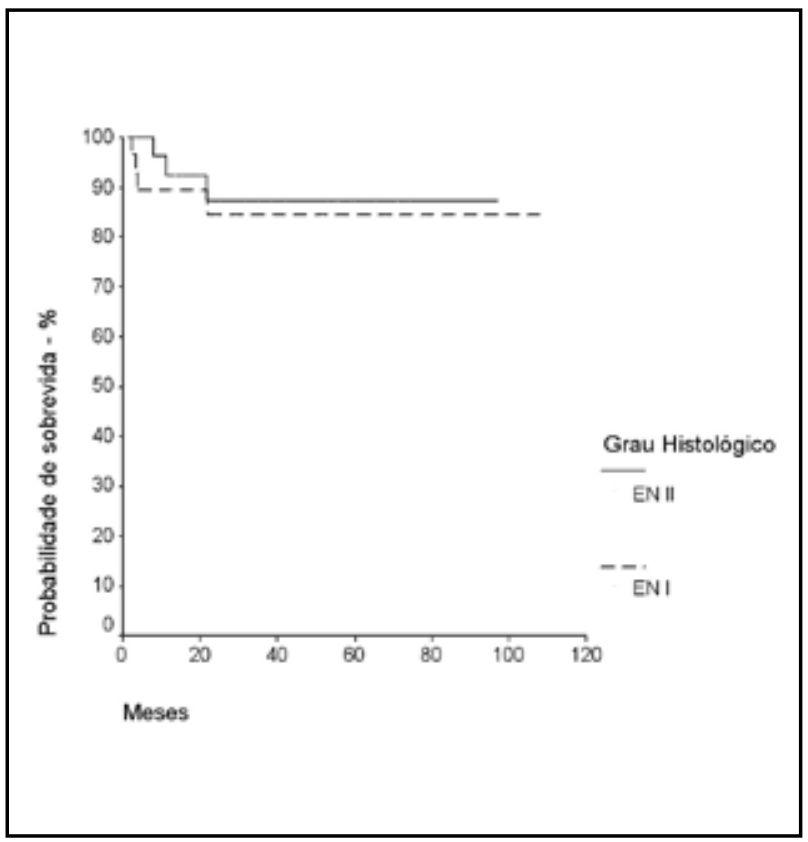

Figura 3 - Sobrevida livre de doença por grau histológico

observaram uma sobrevida livre de doença menor em pacientes portadores de LH EN II, em um estudo que incluiu pacientes tratados com diversas modalidades terapêuticas durante as décadas de 70 e 80 . Portanto, pode ter causado distorção dos resultados obtidos.

Nossos dados sugerem que a graduação histológica proposta pelo BNLI não é preditora do prognóstico de portadores de LH EN tratados com quimioterapia exclusiva ou terapia combinada. A ausência de valor prognóstico da graduação manteve-se mesmo após a exclusão dos pacientes sem avaliação imuno-histoquímica. Deve-se ressaltar, entretanto, que devido à natureza retrospectiva deste estudo e ao pequeno número de pacientes incluídos, é fundamental que essas observações sejam confirmadas por outros estudos brasileiros, preferencialmente de caráter multicêntrico, o que somente será possível com a criação de grupos cooperativos nacionais para o estudo do LH. Todavia, os resultados aqui apresentados são similares aos obtidos em estudos internacionais realizados a partir do início da década de $90^{(3,15,27)}$, os quais demonstram que, em decorrência da efetividade da terapêutica disponível atualmente, o sistema de graduação do BNLI é insuficiente para discriminar o prognóstico do LH EN. Embora seja questionável o valor prognóstico dessa graduação, o reconhecimento dos diferentes graus do LH EN é certamente importante para o patologista, pois, morfologicamente, o LH EN II comporta diagnostico diferencial com outros linfomas não-Hodgkin e, eventualmente, com outras neo- 


\section{Referências}

I. BENNETT, M. H. et al. Analysis of grade I Hodgkin's disease (Report no 6). Clin Radiol, v. 32, p. 49I-8, 1981.

2. BONADONNA, G. et al. Combination chemotherapy of Hodgkin's disease with adriamycin, bleomycin, vinblastine and imidazole carboximide versus MOPP. Cancer, v. 36, p. 252-9, 1975.

3. BOSQ, J. et al. Place of histology in the diagnosis and treatment of Hodgkin's lymphoma: the EORTC-GELA experience. Leukemia Lymphoma, v. 42, p. 47, 2001 . Supplement [Abstract].

4. CARBONE, A. Histologic subclassification of nodular sclerosis Hodgkin's disease. Tumori, v. 65, p. 743-5 I, 1979.

5. ClONINI, L. Et al. Prognostic significance of histologic subdivision of Hodgkin's disease nodular sclerosis. Acta Radiol Oncol, v. 17, p. 65-73, 1977.

6. DEVITA, V.T. Jr. et al. Combination chemotherapy for advanced Hodgkin's disease. Ann Intern Med, v. 73, p. 88I -5, 1970.

7. DORFMAN, R. F. Relationship of histology to site in Hodgkin's disease. Cancer Res, v. 31, p. 1786-9, 1971.

8. DUGGAN, D. B. et al. Randomized comparison of ABVD and MOPP/ABV hybrid for the treatment of advanced Hodgkin's disease: report of an intergroup trial. J Clin Oncol, v. 21, p. 607-| 14, 2003.

9. FERRY, J. A. et al. Hodgkin disease, nodular sclerosis type. Implications of histologic classification. Cancer, v. 7I, p. 45763, 1993.

10. FRANKLIN, J. et al. Is the international prognostic score for advanced stage Hodgkin's disease applicable to early stage patients? Ann Oncol, v. I I, p. 617-23, 2000.

I I. GLICK, J. H. et al. MOPP/ABV hybrid chemotherapy for advanced Hodgkin's disease significantly improves failure-free and overall survival: the 8-year results of the intergroup trial. J Clin Oncol, v. 16, p. 19-26, 1998.

12. HASENCLEVER, D. et al. For the International Prognostic Project on Advanced Hodgkin's Disease: A prognostic score for advanced Hodgkin's disease. N Engl J_OU, v. 339 , p. | 506- |4, 1998.

13. HESS, J. L. et al. Histopathologic grading of nodular sclerosis Hodgkin's disease. Lack of prognostic significance in 254 surgically staged patients. Cancer, v. 74, p. 708- I4, 1994.

14. JAIRAM, R. et al. Histological subclassification of the nodular sclerotic type of Hodgkin's disease. Neth J Med, v. 33, p. 160-7, 1988.
15. KAPLAN, E. L. Et al. Nonparametric estimation from incomplete observations. J Am Stat Assoc, v. 53, p. 457-81, 1958.

16. KLIMO, P. Et al. MOPP/ABV hybrid program: combination chemotherapy based on early introduction of seven effective drugs for advanced Hodgkin's disease. I Clin Oncol, v. 3, p. I 174-82, 1985.

17. LISTER, T. A. et al. Report of a committee convened to discuss the evaluation and staging of patients with Hodgkin disease. J Clin Oncol, v. 7, p. 1630-6, 1898.

18. LUKES, R. J. Et al. Natural history of Hodgkin's disease as related to its pathologic picture. Cancer, v. 19, p. 317-45, 1966.

19. MACLENNAN, K. A. et al. Relationship of histopathologic features to survival and relapse in nodular sclerosing Hodgkin's disease. Cancer, v. 64, p. 1686-93, 1989.

20. MANTEL, N. Evaluation of survival data and two new rank order statistics arising in its consideration. Cancer Chemother Rep, v. 50, p. 163-70, 1966.

2I. MASIH, A. S. Et al. Histologic grade does not predict prognosis in optimally treated, advanced-stage nodular sclerosing Hodgkin's disease. Cancer, v. 69, p. 228-32, 1992.

22. OLIVEIRA, E. D. et al. Hodgkin disease in adult and juvenile groups from two different geographic regions in Brazil: characterization of clinicopathologic aspects and relationship with Epstein-Barr virus infection. Am J Clin Pathol, v. I I8, p. 25-30, 2002.

23. PATCHEFSKY, A. S. et al. Hodgkin's disease: a clinical and pathologic study of 235 cases. Cancer, v. 32, p. 150-61, 1973.

24. SOUZA, C.A. Et al. Hodgkin's disease in Brazil:a clinicopathologic study [Letter]. Haematologica, v. 82, p. 127-8, 1997.

25. SPECTOR, N. et al. C-MOPP/ABV yields good results in a public hospital population with Hodgkin disease in Brazil. Cancer, v. 7I, p. 2823-27, 1993.

26. VAN SPRONSEN, D. J. et al. Disappearance of prognostic significance of histopathological grading of nodular sclerosing Hodgkin's disease for unselected patients, 1972-92. Br J Haematol, v. 96, p. 322-7, 1997.

27. VON WASIELEWSKI, S. et al. Nodular sclerosing Hodgkin's disease: new grading predicts prognosis in intermediate and advanced stages. Blood, v. I O I, p. 4063-9, 2003.

28.WIJLHUIZEN,T. J. et al. Grades of nodular sclerosis in Hodgkin's disease: are they of independent prognostic value? Cancer, v. 63, p. II 50-3, 1989. 